\title{
Severity and Treatment of Alcohol Withdrawal in Elderly Versus Younger Patients
}

\author{
Kirk J. Brower, Sharon Mudd, Frederic C. Blow, James P. Young, and Elizabeth M. Hill
}

\begin{abstract}
We conducted a retrospective chart review of older ( $n=48$; mean age $=69$ ) and younger $(n=36$; mean age $=30)$ patients who were admitted to residential/inpatient treatment for alcohol withdrawal and dependence. Although the two age groups did not differ in terms of recent drinking history, the elderly group had significantly more withdrawal symptoms for a longer duration than the younger group. The elderly group also had more symptoms of cognitive impairment, daytime sleepiness, weakness, and high blood pressure. Finally, no significant differences were found between age groups in either the dosage or number of days of detoxification medication, although a trend was found for more days of medication in the elderly. We conclude that alcohol withdrawal may be more severe in elderly than in younger persons. Accordingly, treatment may take longer and should target the specific profile of symptoms that characterize alcohol withdrawal in the elderly.

Key Words: Withdrawal Syndrome, Alcoholism, Elderly, Drug Therapy, Residential Treatment.
\end{abstract}

$\mathbf{V}$ ERY LITTLE IS known about alcohol withdrawal in elderly patients. A recent literature search revealed only one specific article. ${ }^{1}$ Liskow et al. ${ }^{1}$ reported that elderly alcoholics in residential treatment had more severe ratings of withdrawal and required higher doses of medication for detoxification, despite consuming less alcohol before admission than younger alcoholics. However, the study sample was from a Veterans Administration hospital and may not represent alcoholic patients from other settings.

Other research indicates that elderly alcoholics are more likely than younger alcoholics to manifest biochemical abnormalities, and they are likely to suffer from concomitant physical and cognitive disorders that could complicate the course of withdrawal. ${ }^{2}$ Indeed, a recent study revealed higher rates of mortality from delirium tremens (DTs) in elderly patients than in younger patients. ${ }^{3}$ Another line of evidence suggests that repeated episodes of alcohol withdrawal result in progressively more severe withdrawals, a phenomenon known as kindling. ${ }^{4}$ If living longer is associated with more episodes of withdrawal,

From the Alcohol Research Center and Department of Psychiatry, University of Michigan Medical School (K.J.B., S.M., F.C.B., J.P.Y., E.M.H.), Ann Arbor, Michigan, and Chelsea Arbor Treatment Center, (K.J.B.), Chelsea, Michigan.

Received for publication October 6, 1992; accepted July 28, 1993

This work was supported by the National Institute on Alcohol Abuse and Alcoholism Grant 1P50AA07378.

Reprint requests: Kirk J. Brower, M.D., University of Michigan Alcohol Research Center, 400 East Eisenhower Parkway, Suite A, Ann Arbor, MI 48104.

Copyright (C) 1994 by The Research Society on Alcoholism. then older alcoholics could have more severe withdrawals because of kindling. Kindling may also underlie withdrawal seizures ${ }^{4,5}$ and cognitive impairment ${ }^{6}$ in patients with alcohol dependence. However, we are unaware of studies that examined which types of withdrawal phenomena, such as seizures or cognitive impairment, are more likely to occur in older versus younger alcoholics. If elderly alcoholics do suffer more commonly from particular types of withdrawal phenomena, then treatment approaches may need to be specialized. Taken together, then, these studies suggest that elderly alcoholics may have severe withdrawal syndromes that require more medication and other types of specialized treatment.

Given the apparent lack of studies comparing the course and treatment of alcohol withdrawal in elderly and younger patients, this study was conducted to test the following hypotheses: (1) elderly alcoholics experience a more severe course of withdrawal in terms of the quantity and duration of withdrawal symptoms; (2) elderly alcoholics are more susceptible than younger alcoholics to particular types of symptoms during withdrawal that are known to be associated with aging - in particular, elderly alcoholics will be more likely than younger alcoholics to manifest insomnia and symptoms of delirium, such as cognitive impairment and hallucinations; and (3) elderly alcoholics require higher doses of medication for detoxification for longer durations than younger alcoholics.

\section{METHODS}

\section{Design}

We conducted a retrospective chart review of patients consecutively discharged from 1 of 2 treatment programs during the period from January 1987 to April 1991. This time period allowed us to compare 35-50 patients/treatment program who met the inclusion and exclusion criteria described. Both treatment programs were located at the same community hospital, and one specialized in the treatment of older adults. Both programs exclusively admitted patients with psychoactive substance use disorders and they provided detoxification and rehabilitation services. The chart review was performed by a research nurse and a research assistant. The two reviewers recorded pertinent information from each medical chart onto a structured form that allowed for easy coding and computer entry. Based on a sample of 10 charts, interrater reliability for the two chart reviewers was $87 \%$.

\section{Subjects}

We compared a younger group (ages $21-35 ; n=36$ ) and an older group (ages 60 and above; $n=48$ ) of alcoholic patients. The disparity in age between the two groups was designed to highlight any differences

Alcohol Clin Exp Res, Vol 18, No 1, 1994: pp 196-201 
while minimizing the number of charts reviewed. In addition to age, entry criteria included both of the following: a discharge diagnosis of alcohol dependence and the administration of a complete course of sedative medication for detoxification. The requirement that a patient completed a course of medication for detoxification allowed us to compare this treatment modality for the two age groups. A complete course was defined as initiating and ending sedative medication for detoxification during the index hospitalization. Because patients were generally medicated on an as-needed basis, a complete course of medication could theoretically range from a single dose on 1 day to multiple doses over 14 days. Fourteen days were chosen as the maximum period of observation for the study, because we reasoned that symptoms or treatments that lasted longer than 2 weeks were unlikely to be caused by acute alcohol withdrawal. Cases were excluded if they met any one of the following criteria: ages 36-59; sedative medication for detoxification was not required ( $n=18$ for the older group and 69 for the younger group); detoxification medication was initiated elsewhere immediately before admission $(n=16)$; last drink was more than 7 days before admission $(n=47)$; discharge occurred before pharmacological detoxification was completed (as in a discharge against medical advice) $(n=16)$; a concurrent discharge diagnosis of opioid, cocaine, or other stimulant dependence $(n=31)$, because of the overlap in withdrawal symptoms; and a positive urine drug screen for opioids, cocaine, or amphetamines, even if not diagnosed with dependence $(n=3)$.

\section{Variables of Interest}

Six major domains of variables were studied: (1) demographics, (2) drinking history, (3) past history of withdrawal symptoms and past treatment history, (4) current medical status, (5) withdrawal symptoms during the reviewed treatment episode, and (6) duration and dosage of medications for detoxification. Age, of course, was the major independent variable, whereas withdrawal symptoms (quantity and duration) and treatment received (duration and dosage of medication) were the major dependent variables. Drinking history, past history, and medical status were studied for their possible influence on the withdrawal course and its treatment. Drinking history included duration of problem drinking, days of drinking in the month before admission, number of drinks/day, and recency of last drink before admission. Past history included prior episodes of DTs, seizures, and hallucinations. Past history also included the number of previous inpatient/residential treatment programs for alcohol dependence, which was found to correlate with seizure prevalence in another study. ${ }^{7}$ Medical status included the results of the medical history, physical, and laboratory exams. Of specific interest was the presence of liver disease, pancreatic disease or other gastrointestinal disorders (gastritis, ulcers, and bleeding), and cardiac disease. Laboratory exam included liver function tests and a complete blood count.

The presence of the following 17 withdrawal symptoms were recorded: anxiety, cognitive impairment (which included disorientation or confusion), depressed mood, diaphoresis, hallucinations, headache, insomnia, daytime sleepiness, irritability, nausea or vomiting, psychomotor agitation, seizures, tremors, weakness, high blood pressure (systolic $>140$ $\mathrm{mm} \mathrm{Hg}$ or diastolic $>90 \mathrm{~mm} \mathrm{Hg}$ ), pulse $>100$, and temperature $>$ $99.6^{\circ} \mathrm{F}$. (Although blood pressure, pulse, temperature, and some of the other withdrawal phenomena described are technically signs and not symptoms, for simplicity we refer to them all as symptoms for the purposes of this study.) Insomnia was coded as present if staff intervened at night with a patient due to problems with sleep. Withdrawal symptoms were recorded on the structured chart review form for each day that their presence was documented in the chart. Symptoms were recorded for the first 14 days of treatment or until the patient was discharged, whichever came first. The last day of hospitalization (up to 14 days) on which each symptom (except for vital signs, insomnia, and daytime sleepiness) occurred was recorded. Each patient's maximum last day was used to calculate the mean duration of the withdrawal syndrome for the group. The total number of documented withdrawal symptoms was also recorded for each patient, with a theoretical maximum of 17 symptoms as listed.
Finally, we recorded the following treatments given for withdrawal symptoms. The duration of pharmacotherapy in days and the total dosage across all days of administration were recorded for a variety of detoxification medications, including chlordiazepoxide, lorazepam, diazepam, phenobarbital, atenolol, phenytoin, and L-tryptophan. Only dosage data for the sedative-hypnotics (chlordiazepoxide, lorazepam, diazepam, and phenobarbital) are presented, because the other medications were given too infrequently to make meaningful comparisons between the two age groups. Doses of sedative-hypnotics were first converted to chlordiazepoxide equivalents and then analyzed both before and after adjusting for weight $(\mathrm{mg} / \mathrm{kg})$. The following conversions were used: $25 \mathrm{mg}$ of chlordiazepoxide $=10 \mathrm{mg}$ diazepam $=1 \mathrm{mg}$ lorazepam $=30 \mathrm{mg}$ phenobarbital.

Differences between the two age groups were analyzed by using $t$ tests for continuous variables. Dichotomous variables were tested with either the $\chi^{2}$ test or the Fisher's exact test if cell sizes were too small. All tests were two-tailed. For individual withdrawal symptoms, we predicted age differences for 3 of 17 symptoms (cognitive impairment, hallucinations, and insomnia). Thus, for the remaining 14 symptoms, age comparisons were considered statistically significant if they were less than the Bonferroni-corrected $\alpha$ level of 0.0036 .

\section{RESULTS}

Table 1 illustrates the demographic differences between the two age groups. As expected, older adults were more likely to be widowed, whereas younger adults were more likely to have never married. Nearly equal percentages of the two age groups were currently married. Older adults were also more likely to be retired and less likely to be working compared with the younger group. Finally, the older adults lived with significantly fewer household members. There were no significant differences between the two groups in terms of gender, race, and education.

In terms of drinking history (Table 2), older adults had been problem drinkers for significantly longer than younger adults (19.2 vs. 10.5 years). However, no differences were found in the number of drinks consumed/day, the number of drinking days in the month before admission, or in the time of last drink before admission. Equal numbers of older $(n=5)$ and younger $(n=5)$ patients had concurrent diagnoses of sedative-hypnotic dependence. Past history did not reveal a significant difference in the number of previous hospitalizations for alcohol dependence, despite the longer duration of problem drinking

Table 1. Demographic Characteristics of Older and Younger Alcoholics

\begin{tabular}{|c|c|c|c|}
\hline Variable & $\begin{array}{c}\text { Older } \\
(n=48)\end{array}$ & $\begin{array}{l}\text { Younger } \\
(n=36)\end{array}$ & $p$ value* \\
\hline Age (years) $†$ & $69.0 \pm 6.5$ & $29.7 \pm 4.4$ & $<0.001$ \\
\hline$\%$ Female & 38 & 25 & NSł \\
\hline$\%$ White & 96 & 94 & NS \\
\hline Marital status & & & $<0.001$ \\
\hline$\%$ Never married & 2 & 53 & \\
\hline$\%$ Married & 35 & 33 & \\
\hline$\%$ Separated/divorced & 23 & 14 & \\
\hline$\%$ Widowed & 40 & 0 & \\
\hline Education (years)† & $12.7 \pm 3.4$ & $12.2 \pm 2.5$ & NS \\
\hline$\%$ Employed & 15 & 78 & $<0.001$ \\
\hline No. in householdt & $1.5 \pm 0.7$ & $2.5 \pm 1.2$ & $<0.001$ \\
\hline
\end{tabular}

* Continuous variables were analyzed by $t$ tests and categorical variables by $x^{2}$ tests.

$\dagger$ Values are expressed as the mean $\pm \mathrm{SD}$.

$\ddagger$ NS, not significant. 
Table 2. Drinking History, Past History, and Medical Status

\begin{tabular}{|c|c|c|c|}
\hline Variables & $\begin{array}{c}\text { Older } \\
(n=48)^{*}\end{array}$ & $\begin{array}{l}\text { Younger } \\
(n=36)^{*}\end{array}$ & $\rho$ valuet \\
\hline \multicolumn{4}{|l|}{ Drinking history } \\
\hline $\begin{array}{l}\text { Duration of problem drinking } \\
\text { (years) }\end{array}$ & $19.2 \pm 15.9$ & $10.5 \pm 6.2$ & 0.004 \\
\hline No. of drinks/day & $17.8 \pm 11.9$ & $21.5 \pm 14.1$ & NS $\ddagger$ \\
\hline Days drank in past month & $26.9 \pm 5.6$ & $24.4 \pm 9.1$ & NS \\
\hline Recency of last drink (days) & $0.7 \pm 1.0$ & $0.6 \pm 0.8$ & NS \\
\hline \multicolumn{4}{|l|}{ Past history } \\
\hline No. of prior hospitalizations & $1.5 \pm 2.7$ & $1.1 \pm 1.2$ & NS \\
\hline Seizures & $14.9 \%$ & $11.1 \%$ & NS \\
\hline DTs & $6.4 \%$ & $0.0 \%$ & NS \\
\hline Hallucinations & $16.7 \%$ & $16.7 \%$ & NS \\
\hline Withdrawal tremors & $87.0 \%$ & $94.3 \%$ & NS \\
\hline \multicolumn{4}{|l|}{ Medical status } \\
\hline Liver disease & $53.2 \%$ & $52.8 \%$ & NS \\
\hline Pancreatic disease & $2.1 \%$ & $0.0 \%$ & $\mathrm{NS}^{\mathrm{s}}$ \\
\hline Other gastrointestinal disorders & $40.4 \%$ & $30.6 \%$ & NS \\
\hline Cardiac disease & $56.2 \%$ & $5.6 \%$ & $<0.001$ \\
\hline Hypertension & $25.0 \%$ & $2.8 \%$ & 0.005 \\
\hline AST (5-42 IU/liter)' & $72.4 \pm 52.9$ & $51.3 \pm 44.4$ & NS \\
\hline ALT (6-50 IU/liter) & $45.0 \pm 32.3$ & $58.3 \pm 77.4$ & NS \\
\hline GGTP* (10-70 IU/liter)' & $102.2 \pm 106.6$ & $93.6 \pm 152.5$ & NS \\
\hline $\begin{array}{l}\text { Mean corpuscular volume (82- } \\
100 \mathrm{fl})^{\prime}\end{array}$ & $100.4 \pm 6.6$ & $94.0 \pm 5.9$ & $<0.001$ \\
\hline $\begin{array}{l}\text { Hematocrit (M: } 40-54 \% ; F: 36- \\
47 \%)^{\prime}\end{array}$ & $40.2 \pm 7.1$ & $46.4 \pm 3.2$ & $<0.001$ \\
\hline $\begin{array}{l}\text { Hemoglobin (M: } 13-17 ; \mathrm{F}: 11- \\
15 \mathrm{~g} / \mathrm{dl})^{\prime}\end{array}$ & $13.9 \pm 1.7$ & $15.8 \pm 1.3$ & $<0.001$ \\
\hline $\begin{array}{l}\text { White blood cell count }(3.5-10 \\
\left.10^{\circ} / \text { liter }\right)^{1}\end{array}$ & $6.8 \pm 2.2$ & $8.8 \pm 3.3$ & 0.003 \\
\hline \multicolumn{4}{|c|}{$\begin{array}{l}\text { - Sample size for some variables may be smaller because of missing data. } \\
\text { t Continuous variables were analyzed by } t \text { tests, categorical variables by } \\
\text { sts, unless otherwise noted. For continuous variables, mean values } \pm \text { SD ar } \\
\text { esented. }\end{array}$} \\
\hline
\end{tabular}

in the older adults. Although higher percentages of older adults had prior episodes of seizures and DTs, these differences were not significant (Table 2). Older adults were significantly more likely to suffer from cardiac disease and hypertension, but no significant differences were found for any of the gastrointestinal disorders. Hematologically, older adults were more likely to exhibit macrocytosis as demonstrated by a mean corpuscular volume of $>100 \mathrm{fL}$. Older adults also had significantly lower hematocrits, hemoglobins, and white blood cell counts, but the mean values for these parameters were still within normal clinical ranges (Table 2).

The overall course of alcohol withdrawal and the frequencies of particular types of withdrawal symptoms are shown in Tables 3 and 4. Overall, elderly alcoholics experienced significantly more withdrawal symptoms $(6.8$ vs. 5.6) for a longer time (9.0 days vs. 6.5 days) than younger alcoholics (Table 3 ). None of the patients had seizures during withdrawal, but each of the other withdrawal symptoms were documented at various frequencies (Table 4). The older group (50\%) was much more likely than the younger group ( $8 \%)$ to suffer from cognitive impairment $\left(\chi^{2}=16.4, d f=1, p<0.001\right)$, weakness $(48 \%$ vs. $\left.8 \% ; \chi^{2}=15.1, d f=1, p<0.001\right)$, and high blood
Table 3. Overall Course and Treatment of Alcohol Withdrawal by Age Group

\begin{tabular}{|c|c|c|c|}
\hline Variables & $\begin{array}{c}\text { Oider } \\
(n=48)^{*}\end{array}$ & $\begin{array}{l}\text { Younger } \\
(n=36)^{*}\end{array}$ & $\rho$ value \\
\hline \multicolumn{4}{|l|}{ Overall course } \\
\hline Total no. of withdrawal symptoms & $6.8 \pm 2.0$ & $5.6 \pm 2.2$ & 0.016 \\
\hline $\begin{array}{l}\text { Last day of symptoms (hospital } \\
\text { day no.) }\end{array}$ & $9.0 \pm 4.3$ & $6.5 \pm 4.2$ & 0.011 \\
\hline \multicolumn{4}{|l|}{ Withdrawal treatment } \\
\hline $\begin{array}{l}\text { Unadjusted chlordiazepoxide dose } \\
\text { (mg) }\end{array}$ & $159.7 \pm 105.7$ & $151.5 \pm 119.4$ & NS $\ddagger$ \\
\hline $\begin{array}{l}\text { Total chlordiazepoxide dose (mg/ } \\
\text { kg) }\end{array}$ & $2.3 \pm 1.6$ & $2.1 \pm 1.6$ & NS \\
\hline $\begin{array}{l}\text { Duration of pharmacotherapy } \\
\text { (days)\& }\end{array}$ & $3.5 \pm 3.5$ & $2.3 \pm 2.4$ & NS \\
\hline \multicolumn{4}{|c|}{$\begin{array}{l}\text { "Sample size for some variables may be smaller because of missing data. } \\
t \text { Mean values } \pm \text { SD are presented. Differences between means were analyzed } \\
\text { by } t \text { tests. } \\
\ddagger \text { NS, not significant. } \\
\text { §epresents the number of days (not the last hospital day) on which medication }\end{array}$} \\
\hline
\end{tabular}

Table 4. Frequency of Alcohol Withdrawal Symptoms by Age Group

\begin{tabular}{|c|c|c|c|}
\hline Variables & $\begin{array}{c}\text { Older } \\
(n=48)^{*}\end{array}$ & $\begin{array}{l}\text { Younger } \\
(n=36)^{*}\end{array}$ & $\rho$ value \\
\hline \multicolumn{4}{|l|}{ Frequency $(\%)$ of specific symptoms } \\
\hline Anxiety & $77 \%$ & $89 \%$ & NS $\ddagger$ \\
\hline Cognitive impairment & $50 \%$ & $8 \%$ & $<0.001$ \\
\hline Depressed mood & $65 \%$ & $50 \%$ & NS \\
\hline Diaphoresis & $35 \%$ & $33 \%$ & NS \\
\hline Hallucinations & $4 \%$ & $6 \%$ & NS \\
\hline Headaches & $4 \%$ & $33 \%$ & $<0.001$ \\
\hline Insomnia & $27 \%$ & $19 \%$ & NS \\
\hline Daytime sleepiness & $43 \%$ & $19 \%$ & 0.026 \\
\hline Irritability & $40 \%$ & $25 \%$ & NS \\
\hline Nausea or vomiting & $12 \%$ & $25 \%$ & NS \\
\hline Psychomotor agitation & $25 \%$ & $22 \%$ & NS \\
\hline Shakes or tremors & $92 \%$ & $86 \%$ & NS \\
\hline Seizures & $0 \%$ & $0 \%$ & NS \\
\hline Weakness & $48 \%$ & $8 \%$ & $<0.001$ \\
\hline High blood pressure & $88 \%$ & $69 \%$ & 0.042 \\
\hline High pulse ( $>100$ beats $/ \mathrm{min}$ ) & $65 \%$ & $67 \%$ & NS \\
\hline High temperature $\left(>99.6^{\circ} \mathrm{F}\right)$ & $8 \%$ & $7 \%$ & NS \\
\hline \multicolumn{4}{|l|}{ Other parameters } \\
\hline Highest systolic pressure (mm Hg) & $167.5 \pm 22.3$ & $144.7 \pm 14.0$ & $<0.001$ \\
\hline Highest diastolic pressure $(\mathrm{mm} \mathrm{Hg})$ & $92.4 \pm 11.7$ & $94.8 \pm 13.2$ & NS \\
\hline Highest pulse (beats/min) & $109.6 \pm 17.5$ & $108.8 \pm 16.4$ & NS \\
\hline Highest temperature $\left({ }^{\circ} \mathrm{F}\right)$ & $98.3 \pm 0.9$ & $98.7 \pm 0.6$ & NS \\
\hline
\end{tabular}

- Sample size for some variables may be smaller because of missing data.

t Continuous variables were analyzed by $t$ tests, categorical variables by $x^{2}$ tests, unless otherwise noted. For continuous variables, mean values \pm so are presented.

$\ddagger$ NS, not significant

$\S$ Either systolic pressure $>\mathbf{1 4 0}$ or diastolic pressure $>90 \mathrm{~mm} \mathrm{Hg}$.

pressure ( $88 \%$ vs. $69 \% ; \chi^{2}=4.2, d f=1, p=0.042$ ). Although high blood pressure did not meet significance after applying the Bonferroni-corrected level of $p=$ 0.0036 , the difference between highest systolic pressures did (Table 4). By contrast, the younger group (33\%) was more likely than the older group $(4 \%)$ to suffer from headaches $\left(\chi^{2}=12.6, d f=1, p<0.001\right)$. A nonsignificant trend existed for more insomnia in the elderly. Elderly alcoholics were more than twice as likely to complain of daytime sleepiness, which was nominally significant $\left(\chi^{2}=\right.$ 5.0, $d f=1, p=0.026$ ). Daytime sleepiness could have reflected the effect of either insomnia or sedation from detoxification medication. To help differentiate these factors, we looked for a correlation between dose of chlordi- 
azepoxide equivalents and duration of daytime sleepiness in the elderly. We did not find a significant correlation $\left(r_{s}\right.$ $=-0.18, p=0.24$ ), suggesting that daytime sleepiness was not a side effect of medication. Likewise, we did not find significant correlations between dosage of sedative medication and either cognitive impairment $\left(r_{s}=0.08, p=\right.$ $0.60)$ or weakness $\left(r_{s}=-0.02, p=0.88\right)$ in the elderly, suggesting that these symptoms were not measuring side effects of medication either.

Both before and after adjusting for weight, elderly alcoholics did not require higher doses of chlordiazepoxide equivalents than younger alcoholics (Table 3). Likewise, the elderly did not require significantly more days of detoxification medication (Table 3). Nevertheless, a nonsignificant trend existed for more days of detoxification medication in the elderly ( 3.5 vs. 2.3 days). It should be noted that the duration of pharmacotherapy refers to the number of days on which medication was given. We did not record the value of the last hospital day on which medication was given.

\section{DISCUSSION}

We found that among alcoholics who underwent pharmacological detoxification in an inpatient/residential setting, the elderly group manifested a larger number of withdrawal symptoms for a longer duration than the younger group. Thus, we found support for our first hypothesis that elderly alcoholics undergo a more severe course of withdrawal than younger alcoholics.

The elderly were also more likely to experience symptoms of cognitive impairment, daytime sleepiness, weakness, and high blood pressure; whereas younger alcoholics were more likely to suffer from headaches during withdrawal. Contrary to our prediction, we did not find that older alcoholics were more likely than younger alcoholics to suffer hallucinations during withdrawal. The greater frequencies of cognitive impairment, daytime sleepiness, weakness, and high blood pressure in the elderly may have reflected underlying medical conditions rather than withdrawal symptoms per se. For example, the greater values for high blood pressure during withdrawal in the elderly group may have reflected the higher prevalence of underlying hypertensive disease in our elderly sample (Table 2). Likewise, cognitive impairment, daytime sleepiness, and weakness in the elderly may have been caused by underlying medical conditions such as cardiac disease, hypertension, and anemia rather than by withdrawal per se. Moreover, elderly individuals in general and elderly alcoholics in particular are more susceptible than younger individuals to develop sleep disorders such as sleep apnea, which can result in daytime sleepiness and cognitive impairment. ${ }^{8,9}$

To examine some of these possibilities, we analyzed whether elderly alcoholics with medical conditions were more likely to suffer from daytime sleepiness, cognitive impairment, or weakness than elderly alcoholics without each of the medical conditions listed in Table 2. We conducted 36 comparisons ( 3 symptoms $\times 12$ medical conditions and lab tests). Only two comparisons were significant (Table 5). Elderly patients with cardiac disease were more likely to suffer cognitive impairment $(63 \%)$ during withdrawal than were elderly patients without cardiac disease $(33 \%)\left(\chi^{2}=4.1, d f=1, p=0.042\right)$. Also, elderly patients with hypertension were more likely to have daytime sleepiness (73\%) than elderly patients without hypertension (33\%) $\left(\chi^{2}=5.3, d f=1, p=0.021\right)$. Although no other comparisons were significant, we were unable to rule out the effects of other conditions that accompany aging such as reduced cardiac output, changes in the sleep-wake cycle, and sleep disorders.

We further reasoned that if symptoms were due to withdrawal that we should be able to demonstrate reversibility, whereas if they were due to underlying medical conditions then they should persist. For two symptoms, cognitive impairment and weakness, data were collected regarding the last hospital day that the symptom occurred. The mean last hospital day for cognitive impairment in the elderly (day $3.7 \pm 4.8$ ) was later than in the younger group (day $0.3 \pm 1.2$ ) (Wilcoxon 2-sample test: $Z=-4.1$, $p<0.001$ ). Likewise, the mean last hospital day for weakness in the elderly (day $1.8 \pm 3.0$ ) was later than in the younger group (day $0.2 \pm 0.9$ ) (Wilcoxon 2-sample test: $Z=-3.7, p<0.001$ ). Most importantly, both cognitive impairment and weakness in the elderly ended sooner than withdrawal symptoms taken together (day 9.0 \pm 4.3) (Table 3). These data, then, demonstrate that cognitive impairment and weakness lasted longer, but were still reversible symptoms in the elderly, which is consistent with acute withdrawal rather than with underlying chronic disease.

Finally, we considered the possibility that cognitive impairment, daytime sleepiness, and weakness were side effects of sedative-hypnotic medication. ${ }^{10,11}$ However, the lack of significant correlations between the dosage of medication and the durations of each of the three symptoms suggests that these symptoms were not simply side effects of detoxification medication. Therefore, we conclude, consistent with our second hypothesis, that elderly alcoholics are more susceptible than younger alcoholics to particular types of symptoms during withdrawal, although we falsely predicted hallucinations and failed to predict high blood pressure. We also conclude that other medical conditions may influence the type of withdrawal symptoms found in the elderly.

We did not find strong support for our third hypothesis. Although the elderly group was treated with detoxification medication for slightly more days than the younger group, the difference was not significant. Also contrary to our prediction, equivalent doses of medication were used in the older and younger groups. The lack of difference in medication dosage despite more withdrawal symptoms in 
Table 5. Withdrawal Symptoms as a Function of Cardiac Disease in the Elderly $(n=48)^{*}$

\begin{tabular}{|c|c|c|c|c|c|c|}
\hline \multirow[b]{2}{*}{ Symptom } & \multicolumn{3}{|c|}{ Cardiac disease } & \multicolumn{3}{|c|}{ Hypertension } \\
\hline & Yes & No & $p$ valuet & Yes & No & $p$ valuet \\
\hline Cognitive impairment & $63.0 \%$ & $33.3 \%$ & 0.042 & $66.7 \%$ & $44.4 \%$ & Nsł \\
\hline Daytime sleepiness & $50.0 \%$ & 33.3 & NS & $72.7 \%$ & $33.3 \%$ & 0.021 \\
\hline Weakness & $55.6 \%$ & $38.1 \%$ & NS & $41.7 \%$ & $50.0 \%$ & NS \\
\hline
\end{tabular}

" Sample size for some variables may be smaller because of missing data.

$\dagger \chi^{2}$ two-tailed tests, $d f=1$.

¥NS, not significant.

the elderly group is at odds with the finding of Liskow et al. ${ }^{1}$ We speculate that the particular types of withdrawal symptoms which the elderly manifested (i.e., cognitive impairment, daytime sleepiness, and weakness) contraindicated higher doses of a sedative-hypnotic. Furthermore, elderly individuals are generally more susceptible than younger individuals to the effects of benzodiazepines, because of altered pharmacokinetics and, perhaps, greater neuroreceptor response in the elderly. ${ }^{10,11} \mathrm{We}$ believe, therefore, that the prescribing physicians curtailed dosages appropriately. We further contend that similar doses for slightly longer durations in the elderly actually signified greater medication needs, because of the known altered pharmacokinetic and pharmacodynamic responses to sedative-hypnotics in the elderly. ${ }^{10,11}$

Some research suggests that the severity of alcohol withdrawal increases with increasing episodes of withdrawal, because of a kindling phenomenon. ${ }^{4}$ Thus, elderly alcoholics might be expected to have a more severe withdrawal course because of a longer history of previous withdrawal episodes. We were unable to quantify precisely the number of previous withdrawal episodes, because of the retrospective nature of the study. As an indirect measure, the number of prior hospitalizations for alcohol treatment was used, which has been found to correlate with seizure prevalence in alcoholics. ${ }^{7}$ The number of prior hospitalizations did not differ significantly between the two age groups even though the elderly alcoholics had been problem drinkers for more than 8 years longer (Table 2). We can only speculate that the younger alcoholics had as many admissions as the elderly because their earlier age of onset was associated with severe social consequences that precipitated hospital admissions. ${ }^{12}$ In any case, we did not find significant correlations between the number of prior hospitalizations and the severity of withdrawal symptoms (total number or duration).

Liskow et al. ${ }^{1}$ reported that elderly (predominantly male) alcoholics at a Veterans Administration hospital had more severe ratings of withdrawal and required higher doses of medication for detoxification. Our study extends the finding of greater withdrawal severity in elderly alcoholics to women and to patients found in a community hospital setting. However, we did not find a difference in dosage between our elderly and younger groups.

The retrospective design of our study limits the conclusions that can be drawn from the data. Withdrawal scales were not used by the two treatment programs to measure the type and severity of symptoms. Thus, the type, quantity, and duration of withdrawal symptoms that we measured were dependent on the quality of documentation in the medical record. Because time-conscious clinicians are most likely to document symptoms only when the symptom requires some clinical action, our data probably represent underestimates of the actual number and durations of withdrawal symptoms. In other words, only the most severe symptoms were likely to be documented. This limitation should apply equally to the two age groups. Consequently, we believe that the absolute values of the withdrawal measures in Tables 3 and 4 are not as meaningful as the differences between groups.

Another limitation of the study concerns the possible differences both in charting and in the tendency to medicate patients between the two treatment units. It is possible that staff on the older adult unit more compulsively recorded withdrawal phenomena than the staff on the other unit did. This would give the appearance of more withdrawal symptoms in the older adult group. It is also possible that the physicians and nurses on the older adult unit were more or less likely to use medications than the other treatment staff did because of differences in treatment orientation or philosophy. Indeed, orders for sedative medication were typically written on an as-needed basis. Thus, nursing staff used their discretion as to how much medication was given. On the other hand, the two units shared the same medical director, who was also the attending physician for most cases studied, and the same clinical director. Although each unit may have developed its own specific programming and "treatment culture," there was enough overlap in clinical and administrative staff to permit comparisons for the purpose of this study. Nevertheless, caution is warranted in the interpretation of our results. A stronger study design would standardize the charting and treatment protocols across units.

In conclusion, our study provides further support that alcohol withdrawal may be more severe in the elderly. Treatment programs and third-party payers should recognize that both the course of alcohol withdrawal and its treatment are likely to be longer in the older adult than in the younger adult. To determine more precisely how much longer symptoms occur, future research should use a prospective design and structured rating scales for measuring withdrawal symptoms. Finally, some withdrawal symptoms occur more commonly in the elderly than in younger adults, such as cognitive impairment, daytime 
sleepiness, weakness, and high blood pressure. Such symptoms may in part reflect underlying medical conditions and may require specialized interventions by medical and nursing staff. Whether benzodiazepines are the optimal pharmacotherapy for alcohol withdrawal when characterized by such symptoms is another subject for further research.

\section{ACKNOWLEDGMENTS}

We thank the staff from Medical Records, the Older Adult Inpatient Program, and the Kresge Residential Program at Chelsea Community Hospital, Chelsea, MI, whose work made this study possible.

\section{REFERENCES}

1. Liskow BI, Rinck C, Campbell J, DeSouza C: Alcohol withdrawal in the elderly. J Stud Alcohol 50:414-421, 1989

2. Hurt RD, Finlayson RE, Morse RM, Davis LJ Jr: Alcoholism in elderly persons: Medical aspects and prognosis in 216 inpatients. Mayo Clin Proc 63:753-760, 1988

3. Feuerlein W, Reiser E: Parameters affecting the course and results of delirium tremens treatment. Acta Psychiatr Scand 73(Suppl. 329): $120-123,1986$
4. Brown ME, Anton RF, Malcolm R, Ballenger JC: Alcohol detoxification and withdrawal seizures: Clinical support for a kindling hypothesis. Biol Psychiatry 23:507-514, 1988

5. van Sweden B, Hoste S: Are complex partial seizures an uncommon withdrawal sign in the elderly? Eur Neurol 27:239-244, 1987

6. Glenn SW, Parsons OA, Sinha R, Stevens L: The effects of repeated withdrawals from alcohol on the memory of male and female alcoholics. Alcohol Alcohol 23:337-342, 1988

7. Lechtenberg R, Worner TM: Relative kindling effects of detoxification and nondetoxification admissions in alcoholics. Alcohol Alcohol 26:221-225, 1991

8. Moran MG, Thompson TL II, Nies AS: Sleep disorders in the elderly. Am J Psychiatry 145:1369-1378, 1988

9. Aldrich MS, Shipley JE, Tandon R, Kroll PD, Brower KJ: Sleep disordered breathing in alcoholics: Association with age. Alcohol Clin Exp Res 17:1179-1183, 1993

10. Closser MH: Benzodiazepines and the elderly: A review of potential problems. J Subst Abuse Treat 8:35-41, 1991

11. Lawlor BA, Sunderland T: Use of benzodiazepines in the elderly, in Roy-Byrne PP, Cowley DS (eds): Benzodiazepines in Clinical Practice: Risks and Benefits. Washington, D.C., American Psychiatric Press, 1991, p 213

12. Irwin M, Schuckit M, Smith TL: Clinical importance of age at onset in type 1 and type 2 primary alcoholics. Arch Gen Psychiatry 47:320-324, 1990 